$88 \%$ of the recurrent status group and for all 5 children with multiple episodes of status epilepticus. The risk was related to the severity of the neurological abnormality, the multiply handicapped children being at highest risk. Fifteen of 16 children with recurrent status epilepticus were being treated with antiepileptic drugs at the time of recurrence. The morbidity and mortality of status epilepticus were low. (Shinnar S et al. Recurrent status epilepticus in children. Ann Neurol June 1992; 31:598-604.) (Correspondence: Dr. Shinnar, Division of Pediatric Neurology, VCP-207, Montefiore Medical Center, 111 E. 210th St., Bronx, NY 10467.)

COMMENT. The authors indicate that status epilepticus in neurologically normal children is by and large an isolated event that should not unduly influence decisions regarding further therapy. The high risk subgroup of neurologically abnormal children with recurrent status epilepticus underscored the need for better treatment strategies.

The more frequent use of neurosurgical treatment of refractory status epilepticus is suggested by Gorman DG et al. in a report from the UCLA Pediatric Epilepsy Research Program, Los Angeles, CA. (Epilepsia May/June 1992; 33:546-549.) The resection of a right frontal focus in a 9 year old boy with left focal motor seizures and status epilepticus was followed by immediate control of the seizures and no seizures for more than 1 year post-operatively while using carbamazepine monotherapy.

\title{
EEG MAPPING AND BECT
}

Sequential topographic EEG mapping performed to differentiate "epileptic" from "non-epileptic" rolandic spikes has shown a characteristic pattern significantly related to classical BECT (benign focal epilepsy of childhood with centro-temporal spikes) in a study at the Department of Clinical Neurophysiology and Department of Child Neurology, University Hospital Utrecht, The Netherlands. There were 2 patterns: 1) stationary potential fields, and 2) non-stationary potential fields. The non-stationary patterns represented by a double spike showed a specific sequence starting with a dipolar field, with the negative pole in the frontal region and the positive pole in the centro-temporal region, and changing to a unipolar or dipolar field, with a negative potential field in the centro-temporal region and a simultaneous positive potential field in the frontal region. This characteristic pattern appeared to be significantly related to classical BECT. (Van der Meij W. et al. Sequential EEG mapping may differentiate "epileptic" from "non-epileptic" rolandic spikes. EEG and Clin Neurophysiol June 1992; 82:408-414.) (Correspondence: Dr. W. Van der Meij, Department of Clinical Neurophysiology, University Hospital Utrecht, P.O. Box 85500, 3508 GA Utrecht (The Netherlands).)

COMMENT. The spike morphology in 40 uncontrolled childhood focal epilepsy patients was quantified by a computer-based technique and analyzed in relationship to epileptic syndromes at the Baylor College of Medicine, Houston, TX. The spikes of patients in the benign focal epilepsy category differed from those in other syndromes; they were 
higher in amplitude, longer in duration, less sharp, and had a higher spike parameter. (Frost JD et al. Epilepsia May/June 1992 33:531536.)

A report of unilateral opercular macrogyria underlying benign childhood epilepsy with centrotemporal rolandic spikes in an 18 year old boy emphasizes the need for MRI in detecting neuronal migration disorders and other structural lesions in cases of apparent idiopathic partial epilepsies. (Ambrosetto G. Epilepsia May/June 1992 33:499503.)

\section{CSF ACTH LEVELS IN INFANTILE SPASMS}

ACTH levels in CSF of 14 infants with massive infantile spasms were significantly lower than those of controls in a study from the University of Southern California and Childrens Hospital of Los Angeles, CA. Differences in cortisol levels between patients and controls were not statistically significant. The authors postulate the involvement of the corticotropinreleasing hormone-ACTH-glucocorticoid regulatory loop in the pathophysiology of infantile spasms. (Baram TZ, et al. Brain-adrenal axis hormones are altered in the CSF of infants with massive infantile spasms. Neurology June 1992; 42:1171-1175.) (Reprints: Dr. Tallie Z. Baram, Division of Neurology (\#82), CHLA, P.O. Box 54700, Los Angeles, CA 90054-0700.)

COMMENT. Further studies of this type should help to determine the respective merits of low dose versus high dose ACTH therapy in infantile spasms (see Progress in Pediatric Neurology, ed Millichap JG. $1991 ; 30-34)$.

\section{ANTICONVULSANT DRUGS}

\section{CHEWABLE VERSUS REGULAR CARBAMAZEPINE}

A comparison of chewable and regular carbamazepine (CBZ tablets) in 44 children receiving chronic CBZ monotherapy is reported from the IWK Children's Hospital, Halifax, Nova Scotia. Weekly levels showed no consistent differences between the 1 month on chewable $\mathrm{CBZ}$ and on regular CBZ. Seizure control and rates of reported side effects were similar in the 2 periods. Chewable CBZ produced higher peak CBZ levels in 5 patients and a similar number had higher peaks with regular CBZ. (Camfield $P$ et al. The pharmacology of chewable versus regular carbamazepine in chronically treated children with epilepsy. Can J Neurol Sci May 1992; 19:204-207.)

COMMENT. Chewable and regular CBZ appear to have similar pharmacokinetics but individual patients may show some differences and those with peak level side effects using one form of $\mathrm{CBZ}$ should receive the alternative form of tablet.

A therapeutic bioequivalency study of brand name versus generic carbamazepine in 40 epileptic patients studied at the Bowman Gray 